\title{
LA FORMACIÓN ENFERMERA EN CUIDADOS AL FINAL DE LA VIDA EN LAS UNIVERSIDADES ESPAÑOLAS
}

\author{
Yajaira Hernández González*
}

\section{RESUMEN}

Existen determinadas competencias que un profesional de la enfermería debe desarrollar y dominar para poder ofrecer unos cuidados de calidad a los pacientes a los que atiende. No solo en el ámbito de los cuidados en general a personas, sino en particular en los cuidados al final de sus vidas. Estas habilidades deben ser aprendidas con una adecuada formación académica básica, que posibilite el dominio en estas áreas y que proporcione, así, la mejor calidad de atención a los pacientes y sus familiares. En el presente artículo nos aproximamos brevemente a la atención cotidiana y habitual desarrollada al cuidar a pacientes al final de sus vidas y al nivel de conocimientos impartidos a los estudiantes de enfermería en los estudios de grado existentes en la actualidad en el territorio español, de modo particular a las asignaturas que tienen relación con los cuidados al final de la vida en dichas universidades. Comparamos, asimismo, los datos de la situación existente en nuestro país hace cinco años y en la actualidad, para ver cuál ha sido su avance, además de examinar la cantidad y calidad de dichos conocimientos y la formación impartida en nuestro país.

Palabras clave: formación enfermera, cuidados paliativos y al final de la vida, grado de enfermería.

\section{NURSING TRAINING IN END-OF-LIFE CARE AT SPANISH UNIVERSITIES}

\section{Abstract}

There are specific competencies that a nurse must develop and master in order to provide quality care to the patients they serve. Not only in the field of care in general but in particular in care at the end of their lives. These skills must be learnt with adequate basic academic training, which will allow mastery in these areas and thus provide the best quality of care for patients and their families. In this article, we briefly approach the daily and regular care developed when caring for patients at the end of their lives and the level of knowledge imparted to nursing students in the undergraduate studies currently existing in Spain, in particular the subjects related to care at the end of life in these universities. We also compare the data on the situation in our country five years ago and at present, to see what progress has been made, in addition to examining the quantity and quality of such knowledge and the training provided in our country.

KEYwORDS: nursing training, palliative and end-of-life care, nursing degree.

DOI: https://doi.org/10.25145/j.cuidar.2020.00.07

Revista CuIDAR, 0; junio 2020, pp. 81-97; ISSN: 2660-4426 


\section{INTRODUCCIÓN}

A lo largo del pasado y el presente siglo se ha experimentado un avance científico bastante significativo en lo que respecta al mundo de las ciencias de la salud. Este hecho nos ha permitido lograr un aumento exponencial de la esperanza de vida y, por tanto, un envejecimiento progresivo de la población de los países desarrollados. Dicho avance ha sido posible gracias a una adecuada formación, en el campo del cuerpo humano y la salud, para así solventar los problemas que pudiesen aparecer y curar las diversas enfermedades y patologías existentes, en la medida de lo posible, otorgando a la población la mejor calidad de vida que se pueda obtener.

A pesar de todo, el objetivo de adquirir una adecuada salud para todas las personas no es del todo realista y entraremos, en algún momento, en la etapa final de nuestras vidas. Por otra parte, el número de enfermos considerados como crónicos se ha visto incrementado exponencialmente. Personas que experimentan cada día un deterioro físico y psicológico progresivo y que requieren grandes necesidades de atención y unos cuidados especiales hasta que el desenlace de su situación sea, irremediablemente, la muerte.

Comprender el proceso del morir y los cuidados al final de la vida requiere profundizar en ciertos conceptos, básicos al menos, para proporcionar una atención adecuada dentro del marco de nuestra actividad como profesionales de la salud. Pero ¿estamos realmente preparados para el proceso de la muerte con todas sus consecuencias? ¿Somos capaces de entender que los procesos que experimenta una persona al final de su vida no son solo físicos, sino también emocionales, espirituales y culturales? Que además del sufrimiento de los pacientes, también la familia está implicada en este proceso... Partimos de la realidad de que al ser personal sanitario, estamos supuestamente preparados para afrontar cualquier tipo de situación en la que se vea comprometida la integridad física de una persona, ya sea en situación de riesgo vital o no, pero ¿estamos preparados física, educativa y mentalmente para afrontar la muerte? ¿Se nos proporcionan unos conocimientos mínimos para hacer frente a estas complejas situaciones? Descubramos la respuesta.

La Sociedad Española de Cuidados Paliativos (SECPAL) define el concepto de «situación de enfermedad terminal» como «la presencia de una enfermedad avanzada, progresiva e incurable, en ausencia de posibilidades razonables de respuesta al tratamiento específico, con la aparición de numerosos problemas físicos, multifactoriales y cambiantes, que generan gran impacto emocional en el paciente, familia y equipo terapéutico como consecuencia de la presencia explícita o no de la muerte y con un pronóstico de vida limitado»"

* Enfermera. E-mail: yajairahg@hotmail.es.

1 Espinar Cid, V. Los cuidados paliativos: Acompañando a las personas al final de la vida. Cuad Bioét XXIII [artículo en Internet] 2012 [citado 6 febrero 2015]; [aprox. 8 p.]. Disponible en http:// www.aebioetica.org/revistas/2012/23/77/169.pdf. 
Una situación de tal complejidad como lo es el proceso al final de la vida origina una fuerte demanda de atención y apoyo emocional, tanto por parte del enfermo como de sus allegados, en la que el miedo y la incertidumbre experimentada en este proceso vital están condicionados por factores de tipo cultural y personal de necesario entendimiento por parte del personal sanitario, ya sea médico, enfermero, auxiliar de enfermería...; cualquier profesional que sea responsable de atender las necesidades de los pacientes, en todos sus ámbitos ${ }^{2}$.

Concretamente, en este artículo nos centraremos en el personal de enfermería y en el nivel de conocimientos que poseen para hacer frente a este tipo de pacientes en particular. Pacientes en la fase final de sus vidas, ya sean o no pacientes oncológicos. Nos centraremos por tanto en los cuidados al final de la vida en todas sus dimensiones.

La enfermera desempeña dentro del equipo de salud un lugar privilegiado, pues, entre otras razones, permanece la mayor cantidad de tiempo junto al paciente y su familia. Su campo de acción abarca todas las etapas de la vida, desde el nacimiento hasta la muerte, adecuándose constantemente a las necesidades de cada grupo. Aunque la visión tradicional de su desarrollo profesional, en los últimos años, siempre ha consistido en la prevención de enfermedades, la promoción de la salud y el cuidado del enfermo recuperable, no debemos olvidarnos nunca de que el enfermo en situación terminal también forma parte de nuestro cometido profesional, junto con todas sus necesidades, cuidados y las de sus allegados, que forman parte directa y esencial del bienestar de estos pacientes durante los últimos días de su vida.

La enfermera procura una mayor calidad de vida para los enfermos con padecimientos crónicos y, junto con el resto del equipo, se esfuerza en aliviar los problemas, facilitar los procesos de adaptación a la situación terminal, adecuando la comunicación paciente-familia-equipo y colaborando con los diferentes recursos implicados. Además, su participación comporta planificar los cuidados desde la individualidad, la continuidad y el contenido multidisciplinar ${ }^{3}$. Pero irealmente se llevan todos estos aspectos a cabo de forma adecuada? ¿Estamos lo suficientemente formados como para salir de una carrera universitaria y emprender nuestro cometido profesional, realizándolo de la manera más adecuada que evite así sufrimientos añadidos? Es evidente que la experiencia la proporciona el tiempo y la dedicación a nuestra profesión, pero ¿poseemos un mínimo de conocimientos suficiente que nos ayuden a hacer frente a las dificultades que nos podamos encontrar en nuestro

2 Junin, M. Rol de enfermería en cuidados paliativos. Primer simposio virtual de dolor, medicina paliativa y avances en farmacología del dolor. [Página en Internet] [citado 17 febrero 2015] [aprox. 4 p.]. Disponible en http://omjhnt.com/file-doctc/1AUS/rol-de-enfermer-a-en-cuidados-paliativos-simposio-virtual.html.

${ }^{3}$ López, N.M., Reyes Miranda, T. y Reyes Nieto, M.E. Ética, bioética y legalidad en los cuidados paliativos; competencia de enfermería. Cancerología 5 [artículo en Internet] 2010 [citado 7 febrero 2015]; [aprox. 8 p.]. Disponible en http://www.incan.org.mx/revistaincan/elementos/documentosPortada/1294868609.pdf. 
camino cuando estemos trabajando con enfermos que requieren de nosotros unos cuidados tan específicos?

Diversos autores han tratado el tema de los cuidados paliativos (CP) y los cuidados al final de la vida en diferentes modelos académicos. Es el caso de las autoras Ruland y Moore, quienes definen su teoría de final de vida tranquilo, en la que incluyen ciertos aspectos que se deben incluir en lo que ellas consideran unos cuidados adecuados en situaciones de terminalidad. Postulan una serie de afirmaciones básicas que se deben cumplir para proporcionar estabilidad al paciente en los momentos finales, tales como un adecuado control de la analgesia que ayude a la persona a no experimentar dolor, controlando y aliviando las molestias físicas y facilitando el reposo y la relajación, además de incluir al paciente y sus allegados en la toma de decisiones y hacerlos partícipes en los cuidados, proporcionando apoyo emocional y siempre inspirando confianza en el desempeño de nuestra profesión, teniendo presente que las experiencias del paciente en estos ámbitos contribuyen a un final tranquilo de vida ${ }^{4}$.

Podríamos definir este postulado como el desempeño correcto de nuestra profesión, pues nos asegura una vida y muerte digna en todo momento, tanto para el paciente como para la familia. Ahí radica el fin de nuestras acciones como profesionales de la salud, proporcionar el confort y bienestar necesario y correcto hasta los momentos finales, con el mayor respeto y adecuada ejecución de nuestras tareas profesionales.

Es por este motivo por el cual el papel de enfermería en la atención de pacientes en fase terminal debe tratar de lograr la mayor autonomía posible del individuo y conservar su dignidad hasta la muerte desde el respeto a su voluntad, cubriendo ciertos aspectos como el desarrollo de una comunicación efectiva y el soporte emocional durante su adaptación a la enfermedad, así como el adecuado control de los síntomas prioritarios para la persona afectada, desde el respeto a sus valores y preferencias al igual que su voluntad en la toma de decisiones. Las personas enfermas y sus familias tienen determinados ideales y valores culturales que les son propios, y los enfermeros deben dispensar cuidados que sean sensibles y coherentes a la cultura y que respeten sus creencias espirituales y religiosas, ofreciendo unos cuidados centrados en todas las dimensiones que conforman a cada individuo, propiciando siempre el máximo grado de bienestar para estos pacientes y evitando el uso de medidas desesperadas como el encarnizamiento terapéutico 5 .

${ }^{4}$ Codorniu, N., Bleda, M., Albuquerque, E. et al. Cuidados enfermeros en cuidados paliativos: Análisis, consensos y retos. Index Enferm [artículo en Internet] 2011 [citado 7 febrero 2015]; [aprox. 11 p.]. Disponible en http://scielo.isciii.es/scielo.php?pid=S1132-12962011000100015\&scrip$\mathrm{t}=$ sci_arttext 20(1-2): 71-75. 5. Raile Alligood M. Modelos y teorías en enfermería. 9. ${ }^{a}$ Ed. Elsevier Espańa; 2018. pp. 753-70.

5 Orbegozo, A. y Wilson Astudillo, A. La enfermería y la filosofía de los cuidados al final de la vida. [Página en Internet]. 2003 [citado 7 febrero 2015]; [aprox. 24 p.]. Disponible en http:// www.paliativossinfronteras.com/upload/publica/cuidados\%20paliativos/enfermeria-paliativa/FILOSOFIA\%20DE\%20LOS\%20C.P.\%20Y\%20ENFERMERIA.pdf. Consejo Internacional de enfer- 
Así mismo, personalizar las necesidades de los pacientes es una prioridad a tener en cuenta por parte de los profesionales de la salud, que permita que el proceso de la muerte pueda seguir su curso natural, encaminado siempre a un final digno y lo más sereno posible. Sin embargo, la práctica diaria no está exenta de dificultades para lograr dicha meta. Por ello, es preciso plantearse como el objetivo principal la necesidad de una capacitación o formación del personal de enfermería que desarrolla su trabajo en dicha disciplina, lo cual puede resolver y mejorar la inadecuada prevención o tratamiento de los síntomas del paciente agónico, los fallos de comunicación entre profesionales y familiares o la insuficiente atención a las necesidades de la misma.

Para ejercer estos cuidados de forma eficaz, los profesionales de enfermería deben contar con una formación básica y adecuada que se centre en todos los aspectos relativos a los CP y cuidados al final de la vida en general. En los últimos años, la situación de los cuidados al final de la vida en España ha experimentado cambios significativos en cuanto a contenidos impartidos; aun así, parece insuficiente el nivel de educación proporcionado en este ámbito a día de hoy. Probablemente, se haya producido un aumento y mejorado la formación de los profesionales dedicados a estos temas con un notable incremento de conocimientos en los programas asistenciales, pero, a pesar de eso, cuando observamos la práctica diaria, contrasta con la escasez de conocimientos y competencias generales desarrollados por parte de los profesionales enfermeros a la hora de enfrentarse a todos los aspectos relacionados con la muerte.

El personal enfermero, por el mero hecho de ser el sanitario que mayor parte del tiempo permanece junto al enfermo, hace frente a una serie de circunstancias y situaciones complejas que se dan a lo largo de su jornada laboral cuando atienden a pacientes en el final de su vida. Es por este motivo por el cual podemos prestar una ayuda muy eficaz en la detección, valoración y el manejo de los síntomas del paciente, con el fin de proporcionar un adecuado control de los mismos, confort y bienestar en los últimos días, así como una muerte digna y tranquila en la medida de lo posible. La familia también debe formar parte integrante del equipo que atiende al paciente, involucrándose en la toma de decisiones y finalmente par-

meras. Declaración de posición del CIE sobre la Fundación de la enfermera que dispensa cuidados a los pacientes moribundos y sus familias. [Página en Internet]. Granada, España. Biblioteca Lascasas 2006 [citado 7 febrero 2015]. Disponible en http://www.index-f.com/lascasas/documentos/ lc0161.pdf. Tejedor Torres, J.C., López de Heredia Goya, J., Herranz Rubia, N. et al. Recomendaciones sobre toma de decisiones y cuidados al final de la vida en neonatología. An Pediatr [artículo en Internet] 2013; [citado 7 febrero 2015]; [aprox. 14 p.]. Disponible en http://apps.elsevier.es/watermark/ctl_servlet?_f=10\&pident_articulo=90192855\&pident_usuario=0\&pcontacti$\mathrm{d}=\&$ pident_revista $=37 \& \mathrm{ty}=102 \&$ accion=L\&origen=elsevier\&web=www.elsevier.es\&lan=es\&fichero=37v78n03a90192855pdf001.pdf. Rodrigues Gomes, A.M. El cuidador y el enfermo en el final de la vida-familia y/o persona significativa. Enferm. glob. [revista en Internet]. 2010 febrero [citado 7 febrero 2015]; (18). Disponible en http://scielo.isciii.es/scielo.php?pid=S1695-61412010000100022\&script=sci_arttext. 
ticipando en los cuidados de una manera activa, ya que ellos serán los cuidadores principales en el ámbito domiciliario ${ }^{6}$.

Los síntomas más relevantes que experimenta la persona cuando su cuerpo comienza a dejar de funcionar correctamente son los físicos. Para muchos pacientes, estos son los más importantes, pues si alivias estos síntomas físicos, estás aliviando en gran medida los psicológicos, ya que muchos vienen derivados del dolor y el malestar que la propia enfermedad les está proporcionando y el hecho de saber que el fin puede estar cerca.

Entre los síntomas físicos más relevantes y habituales, obviamente el más importante es el dolor, que en ocasiones se acompaña de otros, no menos importantes, tales como los síntomas gastrointestinales, neurológicos, respiratorios, etc., derivados de la propia enfermedad y de los tratamientos prescritos para paliar la enfermedad. Estos derivan en ocasiones en síntomas psicológicos, bastante profundos para el paciente y la familia, ya que la persona se ve afectada al ver amenazada su condición vital. Pudiendo desarrollar un cuadro de ansiedad, preocupación y miedo y que tienda a pensar más profundamente sobre su vida y el significado de la misma. Así pues, los problemas físicos, psíquicos o espirituales no resueltos pueden causar o exacerbar el sufrimiento, por lo que en una primera etapa es preciso hacer todo lo que esté en nuestras manos para aliviar las causas físicas de tal sufrimiento, pues a medida que la enfermedad evoluciona, los objetivos terapéuticos pasan de ser curativos a centrarse en proporcionar la mejor calidad de vida y, en los momentos finales, la mejor calidad de muerte posible 7 . Llegados a este punto, debemos darnos cuenta de cuándo hemos pasado del umbral curativo al paliativo. Del hecho de poder remitir a una enfermedad a observar que es definitiva y no se

${ }^{6}$ Orbegozo, A., Wilson Astudillo A. La enfermería y la filosofía de los cuidados al final de la vida. [Página en Internet]. 2003 [citado 7 febrero 2015]; [aprox. 24 p.]. Disponible en http://www. paliativossinfronteras.com/upload/publica/cuidados $\% 20$ paliativos/enfermeria-paliativa/FILOSOFIA\%20DE\%20LOS\%20C.P.\%20Y\%20ENFERMERIA.pdf. Priego, R., SÁnchez, L., RodríGUEZ, E., et al. Cuidados paliativos en oncología pediátrica: ¿Q ué puede aportar la enfermería? XII Congreso nacional. Sociedad española de enfermería oncológica [Página en Internet]. Madrid, España. 2009 [citado 7 febrero 2015]. Disponible en http://congreso2009.seeo.org/pages/comunicaciones/ ponencias/232.pdf.

${ }^{7}$ Orbegozo, A., Wilson Astudillo A. La enfermería y la filosofía de los cuidados al final de la vida. [Página en Internet]. 2003 [citado 7 febrero 2015]; [aprox. 24 p.]. Disponible en http:// www.paliativossinfronteras.com/upload/publica/cuidados\%20paliativos/enfermeria-paliativa/FILOSOFIA\%20DE\%20LOS\%20C.P.\%20Y\%20ENFERMERIA.pdf. TeJedor Torres, J.C., LópeZ de Heredia Goya, J., Herranz Rubia, N. et al. Recomendaciones sobre toma de decisiones y cuidados al final de la vida en neonatología. An Pediatr [artículo en Internet] 2013; [citado 7 febrero 2015]; [aprox. 14 p.]. Disponible en http://apps.elsevier.es/watermark/ctl_servlet?_f=10\&pident_ articulo $=90192855 \&$ pident $\_u s u a r i o=0 \&$ pcontactid $=\&$ pident $\_$revista $=37 \&$ ty $=102 \&$ accion $=$ L\&origen=elsevier\&web=www.elsevier.es\&lan=es\&fichero=37v78n03a90192855pdf001.pdf. Priego, R., SÁnchez, L., Rodríguez, E., et al. Cuidados paliativos en oncología pediátrica: ¿̨ué puede aportar la enfermería? XII Congreso nacional. Sociedad española de enfermería oncológica [Página en Internet]. Madrid, España. 2009 [citado 7 febrero 2015]. Disponible en http://congreso2009.seeo.org/ pages/comunicaciones/ponencias/232.pdf. 
puede lograr su remisión con ningún tratamiento. Es ahí cuando pasamos a otro plano en el que debemos enfocarnos, en la medida de lo posible, en evitar causar un sufrimiento añadido innecesario al paciente y familia y facilitar un final digno, lleno del máximo confort y bienestar, libre de sufrimientos al paciente. Librarlo, en definitiva, del encarnizamiento terapéutico, lo que significa, no solo aceptar la opinión y derechos del paciente a elegir las acciones que desea que llevemos con su persona, sino aceptar sus decisiones y las de sus allegados a elegir el mejor camino a seguir, así como actuar conforme a ellas, reconociendo sus valoraciones, decisiones, su dimensión espiritual, cultural y religiosa, en caso de que las tuvieran, y prefirieran detener cualquier tipo de acción que, en definitiva, pudiese provocarles más sufrimiento añadido al que ya poseen.

Esta noción de aceptación de estas acciones puede resultar muy difícil de entender para el personal sanitario, al que se le ha enseñado que debe hacer todo lo posible por salvaguardar la vida de sus pacientes. Pero no toda nuestra actividad enfermera está enfocada a la idea de salvar vidas, sino de cuidar de aquella persona que lo necesite, sea en el ámbito que sea, en todas sus dimensiones como ser humano, física, emocional, cultural... Este desconocimiento de dichas dimensiones, este empeño en solo centrarnos en el aspecto físico del enfermo, puede llevar a los profesionales de la salud, irremediablemente, a sufrir de manera personal, afectando a su salud física y mental, llegando al punto, incluso, de ser incapaces de desarrollar correctamente su cometido dentro de la profesión, llevándonos a un estado de estrés muy característico que puede hacer que lleguemos a detestar nuestra profesión. Es por ello por lo que los profesionales también deben desarrollar mecanismos de protección individual y en equipo para superar estas situaciones, pues aunque sean ellos los profesionales, la mera condición humana influye terriblemente en la mente y bienestar de aquellos que ayudan al necesitado ${ }^{8}$.

Al mismo tiempo que tratamos el control de los síntomas físicos, también debemos atender la parte emocional y espiritual de nuestros pacientes, independientemente de sus creencias religiosas. Sin duda la etapa más importante y decisiva en la vida de toda persona es el fallecimiento de un ser querido. Esta experiencia suscita una serie de reacciones en la familia que pueden condicionar su futuro, pues es considerada como la sensación más profunda y traumática que un ser humano debe afrontar. Su adecuado acompańamiento es un deber del equipo de salud, y es una parte fundamental que nosotros, como profesionales de la salud, también debemos aprender a manejar estas situaciones para evitar sufrimiento ańadido tanto a la

${ }^{8}$ Orbegozo, A., Wilson Astudillo A. La enfermería y la filosofía de los cuidados al final de la vida. [Página en Internet]. 2003 [citado 7 febrero 2015]; [aprox. 24 p.]. Disponible en http:// www.paliativossinfronteras.com/upload/publica/cuidados\%20paliativos/enfermeria-paliativa/FILOSOFIA\%20DE\%20LOS\%20C.P.\%20Y\%20ENFERMERIA.pdf. TIzón BouZA, E. y VÁzQUEZ Torrado, R. Enfermería en cuidados paliativos. Hospitalización durante los últimos días de vida. Enferm Glob [artículo en Internet] 2004 Nov [citado 7 febrero 2015]; [aprox 32 p.]. Disponible en http://dialnet.unirioja.es/servlet/ejemplar?codigo=102576. 
familia y al paciente como a nosotros mismos ${ }^{9}$. La agonía del paciente en fase terminal, el estado de sedación profunda para evitar sufrimientos, el fallecimiento, el duelo de la familia y su adecuado apoyo son aspectos que debemos tratar también durante el cuidado de los pacientes en su etapa final de la vida.

Por todo esto, asistir la muerte de un paciente no debería tratarse solo como un acto médico más, pues exige de los trabajadores sanitarios recurrir a las más elevadas competencias profesionales y profundos dones humanos para otorgar paz y tranquilidad de forma que se pueda afrontar el periodo de duelo de la forma menos traumática posible, sin duda proporcionando nuestro apoyo incluso una vez producido el fallecimiento, puesto que no tratamos solo a nuestros pacientes, también debemos procurar la salud y bienestar de los cuidadores principales ${ }^{10}$.

Por este motivo, la asistencia al paciente y su familia durante este proceso es una tarea que exige del equipo asistencial una profunda implicación y dedicación, así como una adecuada formación y preparación para ser capaces de cuidar a las personas que se encuentran en estas condiciones. Es por ello por lo que, en 2015, se consideró, en la revisión de este trabajo de final de grado, que la formación de los estudiantes de enfermería en temas relativos a los cuidados paliativos y cuidados al final de la vida era imprescindible para que en un futuro estuviesen preparados para afrontar dichas situaciones. Por consiguiente, se llevó a cabo una consulta de todos los planes de estudio del grado de enfermería de todas las universidades españolas, tanto públicas como privadas, y sus respectivas escuelas de enfermería adscritas, para identificar aquellas asignaturas centradas en los CP, cuidados terminales, cuidados

9 Astudillo, W., Orbegozo, A., Díaz-Albo, E. y Bilbao, P. Los cuidados paliativos, una labor de todos. 1. ${ }^{a}$ ed. San Sebastián: Sociedad Vasca de cuidados paliativos; 2007. Bernadé, M., Dall' Orso, P., González, E., la Pera, V., Carrerou, R., Bellora, R., et al. Cuidados paliativos pediátricos. Arch Pediatr Urug. [artículo en Internet]. 2012; [citado 7 febrero 2015]; 83(3): 203210 [aprox. 8 p.]. Disponible en http://www.cuidadospaliativos.org/uploads/2012/11/CP\%20Pediatricos\%20-\%20Uruguay\%202012.pdf.

${ }^{10}$ Raile Alligood, M. Modelos y teorias en enfermería. 9a Ed. Elsevier España; 2018. pp. 753-70. Orbegozo, A., Wilson Astudillo A. La enfermería y la filosofía de los cuidados al final de la vida. [Página en Internet]. 2003 [citado 7 febrero 2015]; [aprox. 24 p.]. Disponible en http://www. paliativossinfronteras.com/upload/publica/cuidados $\% 20$ paliativos/enfermeria-paliativa/FILOSOFIA\%20DE\%20LOS\%20C.P.\%20Y\%20ENFERMERIA.pdf. TejEDOR TorRES, J.C., LópEZ DE Heredia Goya, J., Herranz Rubia, N. et al. Recomendaciones sobre toma de decisiones y cuidados al final de la vida en neonatología. An Pediatr [artículo en Internet] 2013; [citado 7 febrero 2015]; [aprox. 14 p.]. Disponible en http://apps.elsevier.es/watermark/ctl_servlet?_f=10\&pident_articulo $=90192855 \&$ pident_usuario $=0 \&$ pcontactid $=$ \&pident_revista $=37 \&$ ty $=102 \&$ accion $=$ L\&origen $=e l-$ sevier\&web=www.elsevier.es\&lan=es\&fichero=37v78n03a90192855pdf001.pdf. Tizón BouzA, E. y VÁzQUEZ ToRRADO, R. Enfermería en cuidados paliativos. Hospitalización durante los últimos días de vida. Enferm Glob [artículo en Internet] 2004 nov [citado 7 febrero 2015]; [Aprox 32 p.]. Disponible en http://dialnet.unirioja.es/servlet/ejemplar?codigo=102576. Astudillo, W., Orbegozo, A., Díaz-Albo, E. y Bilbao, P. Los cuidados paliativos, una labor de todos. 1. ${ }^{a}$ ed. San Sebastián: Sociedad Vasca de cuidados paliativos; 2007. Bernadá, M., Dall' Orso, P., González, E., La Pera, V., Carrerou, R., Bellora, R., et al. Cuidados paliativos pediátricos. Arch Pediatr Urug. [artículo en Internet]. 2012; [citado 7 febrero 2015]; 83(3): 203-210 [aprox. 8 p.]. Disponible en http:// www.cuidadospaliativos.org/uploads/2012/11/CP\%20Pediatricos\%20-\%20Uruguay\%202012.pdf. 
al final de la vida, etc., ofrecidas en dichos planes de estudio para conocer en qué porcentaje estos conocimientos eran impartidos a los estudiantes en nuestro país, sabiendo así si era suficiente la formación impartida a los mismos en este campo o si, por el contrario, era preciso insistir más profundamente en esta rama de conocimientos para proporcionar los cuidados adecuados que estos pacientes se merecen.

Estos motivos me llevaron en 2015 a realizar una revisión en profundidad de los planes de estudio académicos de las universidades españolas para conocer en qué medida se imparten conocimientos adecuados a los estudiantes de enfermería en los estudios de grado y postgrado, como parte de un trabajo de fin de grado titulado Formación enfermera en cuidados al final de la vida en España: Una revisión. Los datos obtenidos fueron significativos y los veremos, resumidos, a continuación, realizando además un estudio comparativo con los datos obtenidos a fecha de 2020. ¿Cómo ha cambiado la formación sobre CP o cuidados al final de la vida en un periodo de apenas cinco años en nuestro país? ¿Ha mejorado o empeorado?

Nuestro objetivo fue y es conocer si es suficiente el nivel de educación impartido a los estudiantes de enfermería sobre los CP y cuidados al final de la vida en general en los estudios universitarios de España.

\section{RESULTADOS}

Se ha realizado, al igual que en el año 2015, una búsqueda exhaustiva por todas las páginas web de las universidades que actualmente están habilitadas en todo el territorio español, tanto públicas como privadas, con sus respectivas facultades, escuelas de enfermería y centros adscritos, en caso de que los hubiese, consultando todos los planes de estudio del actual grado de enfermería para determinar en cuántas universidades se imparten asignaturas relacionadas con los cuidados paliativos o cuidados al final de la vida a los estudiantes de enfermería. Para posteriormente conocer sus contenidos tras revisar cada guía docente de las asignaturas que imparten dichos contenidos y en qué curso formativo, si son de carácter obligatorio u optativo y, en definitiva, en qué aspectos se basan sus contenidos. Comparamos posteriormente los datos obtenidos hace cinco ańos con los actuales y determinamos la variación producida en este tiempo.

Los datos obtenidos en 2015 en este TFG fueron los siguientes:

Existían distintas universidades españolas que proporcionan en sus planes de estudio asignaturas acerca de la formación en cuidados paliativos o relacionadas con el final de la vida, y en menor medida estudios de máster (títulos propios) que se centran más específicamente en este tema. Se obtuvo que de un total de 107 escuelas y centros de enfermería existentes en 2015 en nuestro país, solo el 35,5\% (38 escuelas) ofrecen en sus programas formativos alguna asignatura referente a los cuidados paliativos o al final de la vida, de las cuales el $75 \%$ de éstas es de carácter obligatorio y el $25 \%$ optativa. Paralelamente, solamente el 9,3\% (10 escuelas) ofertan estudios de posgrado, concretamente másteres. Estos últimos son todos títulos propios. 
Tras consultar las guías docentes de las asignaturas relativas a dichos cuidados nos encontramos con que sus contenidos podemos agruparlos del siguiente modo:

De las 38 escuelas de enfermería españolas que ofertan contenidos en cuidados al final de la vida, solo el $68,5 \%$ de las mismas ofertan conocimientos acerca del control de síntomas a pacientes en fase terminal. Asimismo, el 51,4\% se centran específicamente en el ámbito del dolor como síntoma principal y lo estudian por separado del resto de síntomas. Seguidamente, también un 51,4\% trata el tema de la agonía y un 54,2\% hablan del duelo. Agrupan estos dos temas que le sirven para abordar el apoyo e integración de la familia en el proceso de los cuidados del paciente, aunque solo el 48,6\% de las escuelas lo toman en consideración. El mismo porcentaje, incluye la importancia de la comunicación como medio de interacción entre familia-paciente y profesional, así como saber comunicar malas noticias, Sólo un $31,4 \%$ de estas 38 escuelas hablan del proceso natural de la muerte y el morir.

Es llamativo en los resultados obtenidos que únicamente un 14,3\% de los programas formativos incluyen contenidos relativos a las necesidades espirituales de los pacientes, un 11,4\% hablan sobre el estrés que genera el desarrollo de estos cuidados para los profesionales que atienden a pacientes terminales y proporcionan pautas y conocimientos para combatir los efectos de los conflictos emocionales que este personal pueda experimentar. Del mismo modo, un $11,4 \%$ de las escuelas forman a sus estudiantes sobre la noción de calidad de vida, el confort y bienestar como elementos necesarios para la satisfacción de los pacientes y familiares durante la etapa final y exclusivamente un $2,9 \%$ tiene presente la importancia de los derechos y voluntades de los mismos.

Una vez realizada una búsqueda idéntica de los planes de estudios de los grados y postgrados de enfermería de las universidades españolas en la actualidad, en el presente año 2020, hemos obtenido que, a día de hoy, existe un total de 76 universidades en España, de las cuales 50 son públicas y 26 privadas. Un total de facultades, escuelas o centros adscritos a las mismas de 120 de las cuales 91 pertenecen a las universidades públicas y 29 a privadas.

De todas estas instituciones, solo 34 universidades de las 76 , un $44,7 \%$, ofertan asignaturas relacionadas con los $\mathrm{CP}$ o cuidados al final de la vida en sus planes de estudio. Dividido por centros, 54 de los mismos, un $45 \%$ ofertan estos estudios en los planes de grado de enfermería. En lo que respecta a las asignaturas impartidas, un $83,3 \%$ de las mismas son de carácter obligatorio y el $16,6 \%$ son optativas, independientemente del curso en el que se quieran realizar (figura 1).

Respecto a estudios postgrado, se ha obtenido que solo tenemos, a fecha de 2020, ocho másteres de enfermería relacionados con esta temática, cinco de ellos son títulos propios y tres oficiales.

Se han cerrado algunas escuelas y estudios presentes en el año 2015, y se han incorporado nuevos estudios de grado en enfermería en otras universidades.

Con respecto a los contenidos hallados dentro de las guías docentes de las asignaturas de las 54 escuelas de enfermería que ofertan contenidos relativos al final de la vida, un 40,7\% trata los contenidos de los síntomas físicos en general (gastrointestinales, respiratorios, etc.) dentro de sus planes de estudio y tan solo un 


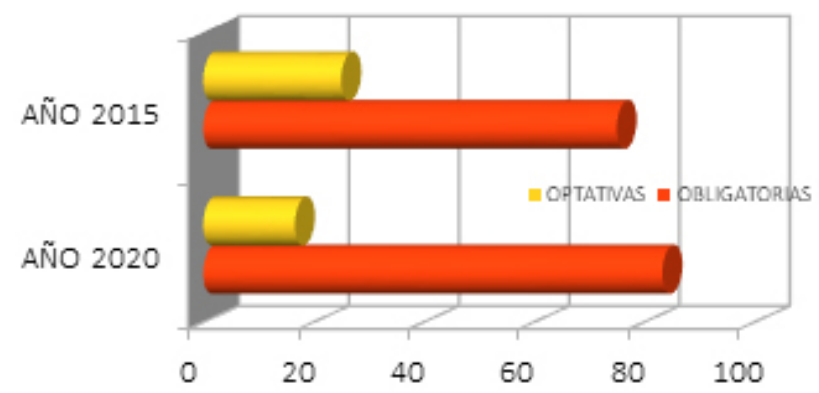

Figura 1: Comparativa entre el año 2015 y 2020 del carácter obligatorio u optativo de las asignaturas impartidas.

$37 \%$ de estos diferencia el síntoma del dolor aparte del resto de los demás, caracterizándolo como el más significativo para los pacientes. En este sentido el 31,4\% abordan la agonía y un 25,9\% su consecuente paliación con sedación y farmacología apropiada. En lo relativo a los aspectos psicológicos y la dimensión espiritual del paciente, podemos decir que los aspectos psicológicos, emocionales y personales son tratados en un $18,5 \%$ de las escuelas; las necesidades de confort, bienestar, calidad de vida en un 16,6\%, la dimensión espiritual, cultural o religiosa, cada vez más presente en nuestras vidas, se trata solo en un $14,4 \%$ de las asignaturas, así como el apoyo familiar, la toma de decisiones, la pérdida y el duelo que se imparte entre un $20-30 \%$ de las asignaturas.

La presencia de un plan de cuidados individualizado, cuidados multidisciplinar con inclusión de todos los miembros del equipo terapéutico se incluye en un $27,7 \%$, que considera este aspecto como relevante en la atención del paciente agónico.

Los datos más significativos encontrados en los planes de estudio actuales han sido que solo un $7,4 \%$ le da importancia a la muerte digna del paciente enfermo, sin sufrimientos añadidos. El mismo porcentaje se dedica al entendimiento del estrés que viven las enfermeras, donde se enseñan técnicas de superación y de afrontamiento.

He encontrado en las guías del curso actual 2019-2020, de algunas facultades (ver anexo), la oferta de información y conocimientos básicos para ser capaces de tratar pacientes oncológicos o terminales pediátricos. De modo que se avanza en la conciencia de la terminalidad en todas las etapas de la vida, aunque de momento este tema solo lo incluyen un $12,9 \%$ del total de los centros que ofertan estudios sobre CP (figura 2). 


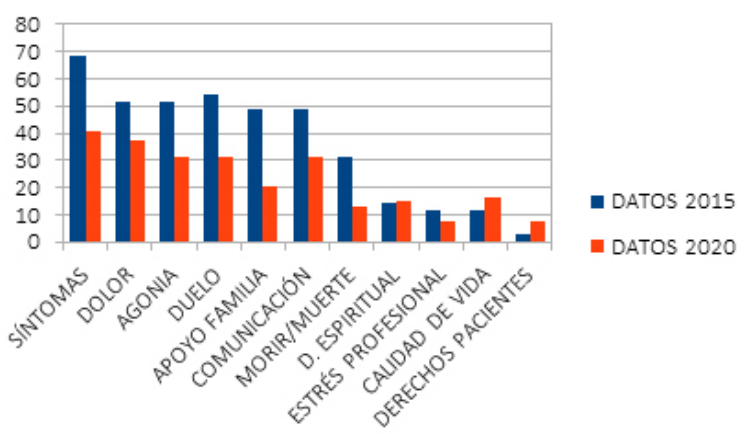

Figura 2. Comparativa entre años 2015 y 2020 de los contenidos impartidos en las guías docentes de las asignaturas pertenecientes al grado de enfermería.

\section{CONCLUSIONES}

Observamos como en el transcurso de un periodo de apenas cinco años, la formación impartida a los estudiantes de enfermería de las universidades españolas acerca de los CP y cuidados al final de la vida tiene un crecimiento significativo, pasando del 35,5\% al 44,7\% (un aumento aproximado del 9\%). Así mismo, también ha aumentado el carácter obligatorio de las asignaturas impartidas llegando a superar en la actualidad el $80 \%$.

La diferencia más significativa que he podido observar en los contenidos de dichas asignaturas es que mientras muchas de ellas han disminuido su cantidad de conocimientos, tales como los síntomas, el dolor, la agonía, el duelo y el apoyo familiar, la comunicación e información de los hechos a pacientes y familiares..., otros temas han aumentado en nivel de especificidad, tales como la concienciación del estrés sufrido por los profesionales de la salud, la importancia de la calidad de vida del paciente y el derecho a una muerte digna, así como todos los demás derechos que envuelven a la persona a elegir sobre su vida o, en estos casos, sobre su muerte.

Se han disminuido conocimientos sobre ciertos aspectos básicos de las materias en muchas escuelas en beneficio de incluir otros nuevos más relevantes y actuales, tales como los aspectos ético-legales de los cuidados terminales, el empleo de la sedación y farmacología adecuada para evitar esa agonía sufrida por los pacientes, la realización de planes de cuidados específicos con trabajo en equipo multidisciplinar que da más importancia a la dimensión psicológica y a los aspectos emocionales que envuelven el proceso de la enfermedad y que, por primera vez, incluyen el tema de los $\mathrm{CP}$ en pacientes pediátricos. Un tema muy importante que no debemos olvidar, como profesionales de la salud que somos, es que los CP y cuidados al final de la vida no solo se presentan en pacientes adultos, también los niños entran en este campo de actuación enfermera. 


\section{REFERENCIAS BIBLIOGRÁFICAS}

Astudillo, W., Orbegozo, A., Díaz-Albo, E. y Bilbao, P. Los cuidados paliativos, una labor de todos. 1. ${ }^{a}$ ed. San Sebastián: Sociedad Vasca de cuidados paliativos; 2007.

Bernadá, M., Dall' Orso, P., González, E., la Pera, V., Carrerou, R., Bellora, R., et al. Cuidados paliativos pediátricos. Arch Pediatr Urug. [artículo en Internet]. 2012; [citado 7 febrero 2015]; 83(3): 203-210 [aprox. 8 p.]. Disponible en http://www.cuidadospaliativos. org/uploads/2012/11/CP\%20Pediatricos\%20-\%20Uruguay\%202012.pdf.

Codorniu, N., Bleda, M., Albuquerque, E. et al. Cuidados enfermeros en cuidados paliativos: Análisis, consensos y retos. Index Enferm [artículo en Internet] 2011 [citado 7 febrero 2015]; [aprox. 11 p.]. Disponible en http://scielo.isciii.es/scielo.php?pid=S1132-12962011000100015\&script=sci_arttext 20(1-2): 71-75.

Consejo Internacional de enfermeras. Declaración de posición del CIE sobre la Fundación de la enfermera que dispensa cuidados a los pacientes moribundos y sus familias. [Página en Internet]. Granada, España. Biblioteca Lascasas 2006 [citado 7 febrero 2015]. Disponible en http://www.index-f.com/lascasas/documentos/lc0161.pdf.

Espinar Cid, V. Los cuidados paliativos: Acompañando a las personas al final de la vida. Cuad Bioét XXIII [artículo en Internet] 2012 [citado 6 febrero 2015]; [aprox. 8 p.]. Disponible en http:// www.aebioetica.org/revistas/2012/23/77/169.pdf.

Junin, M. Rol de enfermería en cuidados paliativos. Primer simposio virtual de dolor, medicina paliativa y avances en farmacología del dolor. [Página en Internet] [citado 17 febrero 2015] [aprox. 4 p.]. Disponible en http://omjhnt.com/file-doctc/1AUS/rol-de-enfermer-a-en-cuidados-paliativos-simposio-virtual.html.

López, N.M., Reyes Miranda T., Reyes Nieto ME. Ética, bioética y legalidad en los cuidados paliativos; competencia de enfermería. Cancerología 5 [artículo en Internet] 2010 [citado 7 febrero 2015]; [aprox. 8 p.]. Disponible en http://www.incan.org.mx/revistaincan/elementos/documentosPortada/1294868609.pdf.

Orbegozo, A., Wilson Astudillo A. La enfermería y la filosofía de los cuidados al final de la vida. [Página en Internet]. 2003 [citado 7 febrero 2015]; [aprox. 24 p.]. Disponible en http:// www.paliativossinfronteras.com/upload/publica/cuidados\%20paliativos/enfermeria-paliativa/FILOSOFIA\%20DE\%20LOS\%20C.P.\%20Y\%20ENFERMERIA.pdf.

Priego, R., SÁnchez, L., Rodriguez, E., et al. Cuidados paliativos en oncología pediátrica: ¿Qué puede aportar la enfermería? XII Congreso nacional. Sociedad española de enfermería oncológica [Página en Internet]. Madrid, Espańa. 2009 [citado 7 febrero 2015]. Disponible en http://congreso2009.seeo.org/pages/comunicaciones/ponencias/232.pdf.

Raile Alligood, M. Modelos y teorias en enfermería. 9. ${ }^{a}$ Ed. Elsevier España; 2018. pp. 753-770.

Rodrigues Gomes, A.M. El cuidador y el enfermo en el final de la vida - familia y/o persona significativa. Enferm. glob. [revista en Internet]. 2010 febrero [citado 7 febrero 2015]; (18). Disponible en http://scielo.isciii.es/scielo.php?pid=S1695-61412010000100022\&script=sci_arttext.

Tejedor Torres, J.C., López de Heredia Goya, J., Herranz Rubia, N. et al. Recomendaciones sobre toma de decisiones y cuidados al final de la vida en neonatología. An Pediatr [artículo en Internet] 2013; [citado 7 febrero 2015]; [aprox. 14 p.]. Disponible en http://apps.elsevier.es/watermark/ctl_servlet?_f=10\&pident_articulo=90192855\&pident_usuario=0\&pcontactid=\&pident_revista=37\&ty=102\&accion=L\&origen=elsevier\&web=www.elsevier. es\&lan=es\&fichero=37v78n03a90192855pdf001.pdf. 
Tizón Bouza, E. y Vázquez Torrado, R. Enfermería en cuidados paliativos. Hospitalización durante los últimos días de vida. Enferm Glob [artículo en Internet] 2004 Nov [citado 7 febrero 2015]; [Aprox 32 p.]. Disponible en http://dialnet.unirioja.es/servlet/ejemplar?co$\underline{\text { digo }=102576}$. 


\section{ANEXO}

Estudios de enfermería. Universidad de Córdoba. [Página en Internet]. Córdoba; 2015. Disponible en http://www.uco.es/enfermeria/grado-enfermeria/planificacion/index.html\#primero [citado 29 febrero 2020].

Universidad Alfonso X El Sabio. Grado en enfermería. [Página en Internet]. Madrid; 2015. Disponible en http://www.uax.es/grado-en-enfermeria.html [citado 29 febrero 2020].

Universidad Autónoma de Barcelona. Grado en enfermería. [Página en Internet]. Barcelona; 2020 [citado 29 febrero 2020]. Disponible en https:/www.uab.cat/web/estudiar/llistat-de-graus/ pla-d-estudis/pla-d-estudis-i-horaris/infermeria-escola-adscrita-sant-cugat-1345467811493. html?param1=1345767862121.

Universidad Católica de Murcia. Grado en enfermería. [Página en Internet]. Murcia; 2015. Disponible en http://www.ucam.edu/estudios/grados/enfermeria-presencial/plan-de-estudios [citado 29 febrero 2020].

Universidad Complutense de Madrid. Grado en enfermería. [Página en Internet]. Madrid; 2015. Disponible en http://www.ucm.es/estudios/grado-enfermeria-estudios-competencias. http:// www.ucm.es/data/cont/docs/titulaciones/26.pdf [citado 29 febrero 2020].

Universidad complutense de Madrid. Grado en enfermería. [Página en Internet]. Madrid; 2020 [citado 29 febrero 2020 ]. Disponible en https:/www.ucm.es/estudios/grado-enfermeria.

Universidad de Alcalá. Grado en enfermería [Página en Internet]. Madrid; 2015. Disponible en http:// www.uah.es/acceso_informacion_academica/primero_segundo_ciclo/matricula_I_II_ ciclo/documentos/Hojas_matricula/G209.pdf [citado 29 febrero 2020].

Universidad de Alcalá. Grado en enfermería [Página en Internet]. Madrid; 2015. Disponible en http:// www.uah.es/estudios/asignaturas/lstAsignaturas_v3.asp?CodCentro $=201 \& \operatorname{CodPlan}=\mathrm{G} 255$ [citado 29 febrero 2020].

Universidad de Alicante. Grado en enfermería [Página en Internet]. Alicante; 2015. Disponible en http://cvnet.cpd.ua.es/webcvnet/planestudio/planestudiond.aspx?plan=C351\# [citado 29 febrero 2020].

Universidad de Cádiz. [Página en Internet]. Cádiz; 2015. Disponible en http://www.uca.es/es/estudios-y-acceso/oferta-general-de-estudios/grados [citado 29 febrero 2020 ].

Universidad de Extremadura. Grado en enfermería [Página en Internet]. Extremadura; 2015. Disponible en http://www.unex.es/conoce-la-uex/centros/cum/titulaciones/grado [citado 29 febrero 2020].

Universidad de Granada. Facultad de enfermería [Página en Internet]. Granada; 2015. Disponible en http://depenfermeria.ugr.es/static/GestorDocencia [citado 29 febrero 2020].

Universidad de Granada. Máster en Cuidados de salud para la promoción de la autonomía de las personas y la atención a los procesos del fin de vida. [Página en Internet]. Granada; 2015. Disponible en http://masteres.ugr.es/cuidados/pages/ficha\#toc_contenido [citado 29 febrero 2020].

Universidad de Huelva. Facultad de enfermería [Página en Internet]. Huelva; 2015. Disponible en http://www.uhu.es/enfe/ [citado 29 febrero 2020.

Universidad de Jaén. [Página en Internet]. Jaén; 2015. Disponible en https://uvirtual.ujaen.es/pub/es/ informacionacademica/catalogoguiasdocentes/p/2014-15/7/100A [citado 29 febrero 2020]. 
Universidad de La Laguna. Máster universitario en cuidados al final de la vida. [Página en Internet]. Tenerife; 2015. Disponible en http://www.departamentos.ull.es/view/titulospropios/tanatologia/Inicio/es [citado 29 febrero 2020].

Universidad de La Rioja. Grado en enfermería [Página en Internet]. La Rioja; 2015. Disponible en http://www.unirioja.es/estudios/grados/enfermeria/guias.shtml [citado 29 febrero 2020].

Universidad de las Islas Baleares. Grado en enfermería. [Página en Internet]. Islas Baleares; 2020 [citado 29 febrero 2020]. Disponible en https://estudis.uib.cat/grau/.

Universidad de León. Grado en enfermería [Página en Internet]. León; 2015. Disponible en http:// www.unileon.es/estudiantes/estudiantes-grado/oferta-de-estudios/planes?titula $=1105$ [citado 29 febrero 2020].

Universidad de Murcia. Escuela universitaria de enfermería de Cartagena. [Página en Internet]. Murcia; 2015. Disponible en http://www.um.es/web/enfermeria-ct/contenido/estudios/grados/ enfermeria/2014-15/guias [citado 29 febrero 2020].

Universidad de Navarra. Grado en enfermería [Página en Internet]. Navarra; 2015. Disponible en http://www.unav.edu/web/grado-en-enfermeria/plan-de-estudios/asignaturas [citado 29 febrero 2020].

Universidad de Oviedo. Grado en enfermería [Página en Internet]. Oviedo; 2015. Disponible en http://www.uniovi.es/estudios/guias/grados/-/asset_publisher/bePFpf8XkIxB/content/ grado-en-enfermeria-centro-adscrito-2013? redirect=\%2Festudios $\% 2$ Fguias $\% 2$ Fgrados [citado 29 febrero 2020].

Universidad de País Vasco. Grado en enfermería. [Página en Internet]. País Vasco; 2020 [citado 29 febrero 2020]. Disponible en https://www.ehu.eus/eu/erizaintzako-gradua-gipuzkoa/kreditu-eta-irakasgaiak-ikasturteka.

Universidad de Salamanca. Escuela universitaria de Ávila [Página en Internet]. Salamanca; 2015. Disponible en http://www.usal.es/webusal/files/Plan\%20Estudios\%20Enfermeria\%20 Avila\%20CAdscrito_modif.pdf [citado 29 febrero 2020].

Universidad de Salamanca. Escuela universitaria de Enfermería de Zamora [Página en Internet]. Salamanca; 2015. Disponible en http://www.usal.es/webusal/node/4556?mst=23http:// www.usal.es/webusal/files/Plan\%20Estudios\%20Enfermeria\%20Zamora\%20CAdscrito_ modif.pdf [citado 29 febrero 2020].

Universidad de Salamanca. Escuela universitaria de Enfermería y fisioterapia. [Página en Internet]. Salamanca; 2015. Disponible en http://enfermeria.usal.es/index.php/titulaciones/gradoenfermeria/plan-de-estudios [citado 29 febrero 2020].

Universidad de Salamanca. Máster en el tratamiento de soporte y cuidados paliativos en el enfermo oncológico. [Página en Internet]. Salamanca; 2015. Disponible en http://www.usal.es/webusal/node/48597 [citado 29 febrero 2020].

Universidad de Salamanca. Máster en tratamiento del dolor [Página en Internet]. Salamanca; 2015. Disponible en http://www.usal.es/webusal/node/40411 [citado 29 febrero 2020].

Universidad de Sevilla. Grado en enfermería [Página en Internet]. Sevilla; 2015. Disponible en http:// www.us.es/estudios/grados/plan_157?p=7 [citado 29 febrero 2020].

Universidad de Vigo. Grado en enfermería. [Página en Internet]. Vigo; 2020 [citado 29 febrero 2020]. Disponible en https://secretaria.uvigo.gal/docnet-nuevo/guia_docent/?consulta=assignatures\&ensenyament=V52G140V01. 
Universidad del País Vasco. Máster especialista universitario en cuidados paliativos. [Página en Internet]. País Vasco; 2015 [citado 29 febrero 2020]. Disponible en http://www.hospital-sanjuandedios.es/cas/master-especialista-universitario-en-cuidados-paliativos.html.

Universidad Pompeu Fabra. Grado en enfermería. [Página en Internet]. Barcelona; 2020 [citado 29 febrero 2020]. Disponible en http://www.tecnocampus.cat/ca/grau/infermeria/pla-estudis.

Universidad Pontificia de Comillas. Escuela universitaria de Enfermería y fisioterapia. [Página en Internet]. Madrid; 2015. Disponible en http://www.euef.upcomillas.es/es/grados/grado-en-enfermeria [citado 29 febrero 2020].

Universidad Pontificia de Comillas. Máster universitario en cuidados paliativos. [Página en Internet]. Madrid; 2015. Disponible en http://www.euef.upcomillas.es/es/postgrado/sanitaria/ master-universitario-en-cuidados-paliativos [citado 29 febrero 2020].

Universidad Pontificia de Salamanca. Grado en enfermería. [Página en Internet]. Salamanca; 2015. Disponible en http://www.upsa.es/estudiar/estudios/titulacion/plan-de-estudios.php?idTit=1011 [citado 29 febrero 2020].

Universidad Pontificia de Salamanca. Máster en cuidados integrales y paliativos en enfermos terminales. Nuevas tecnologías. [Página en Internet]. Salamanca; 2015. Disponible en http://www. upsa.es/estudiar/estudios/titulacion/posgrado/ficha.php?idTit=6U [citado 29 febrero 2020].

Universidad Rey Juan Carlos. Grado en enfermería. [Página en Internet]. Madrid; 2020 [citado 29 febrero 2020]. Disponible en https://www.urjc.es/estudios/grado/590-enfermeria\#itinerario-formativo.

Universidat Rovira i Virgili. Grado en enfermería [Página en Internet]. Tarragona; 2015. Disponible en http://moodle.urv.cat/docnet/guia_docent/index.php?centre=18\&ensenyament=1820\&consulta=assignatures [citado 29 febrero 2020].

Universitat Autónoma de Barcelona. Escola Universitària d'Infermeria Sant Pau. [Página en Internet]. Barcelona; 2015. Disponible en http://webs01.santpau.cat/polymitaImages/public/ EUI-web/Estudis/Pla\%20estudis\%20GI/Pla_Estudis_GI.pdf [citado 29 febrero 2020].

Universitat Autónoma de Barcelona. Máster en Infermeria oncológica. [Página en Internet]. Barcelona; 2015 [citado 29 febrero 2020]. Disponible en http://webs01.santpau.cat/collage/ images/potter/tempFiles/potterTmpFile_uuid_188092/14-15\%2BInfermeria\%2BOncol\%25C3\%25B2gica.pdf.

Universitat Autónoma de Madrid. Máster en Cuidados Paliativos y Tratamiento de Soporte del Enfermo con Cáncer [Página en Internet]. Madrid; 2015. Disponible en http://www.uam. es/ss/Satellite/es/1242654675830/1242656616884/estudiopropio/estudioPropio/Master_ en_Cuidados_Paliativos_y_Tratamiento_de_Soporte_del_Enfermo_con_Cancer.htm [citado 29 febrero 2020].

Universitat de Barcelona. Escola Universitària d'Infermeria. [Página en Internet]. Barcelona; 2015. Disponible en http://eui.hsjdbcn.org/grau-en-infermeria/pla-destudis [citado 29 febrero 2020].

Universitat de Lleida. Facultat d'Infermeria [Página en Internet]. Lleida; 2015. Disponible en http:// www.infermeria.udl.cat/ca/pla-formatiu/pla-estudis-guies-docents.html [citado 29 febrero 2020].

Universitat de Vic. Universitat central de Catalunya. Grado en enfermería [Página en Internet]. Catalunya; 2015. Disponible en http://www.uvic.es/estudi/infermeria [citado 29 febrero 2020].

Universitat Jaume I de Castellón. Grado en enfermería. [Página en Internet]. Comunidad Valenciana; 2020 [citado 29 febrero 2020]. Disponible en https://www.uji.es/estudis/oferta/base/ graus/2019/infermeria. 
